\title{
Anisotropic non-Gaussianity from vector field perturbations
}

\author{
Mindaugas Karčiauskas, ${ }^{*}$ Konstantinos Dimopoulos, ${ }^{\dagger}$ and David H. Lyth ${ }^{\ddagger}$ \\ Department of Physics, Lancaster University, Lancaster LA1 4YB, United Kingdom \\ (Received 26 May 2009; published 14 July 2009)
}

\begin{abstract}
We suppose that a vector field perturbation causes part of the primordial curvature perturbation. The non-Gaussianity parameter $f_{\mathrm{NL}}$ is then, in general, statistically anisotropic. We calculate its form and magnitude in the curvaton scenario and in the end-of-inflation scenario. We show that this anisotropy could easily be observable.
\end{abstract}

DOI: 10.1103/PhysRevD.80.023509

PACS numbers: $98.80 . \mathrm{Cq}$

\section{INTRODUCTION}

The primordial curvature perturbation $\zeta$ provides one of the few windows available to the early Universe, and is the subject of intense interest at present. It may be that $\zeta$ is practically Gaussian. Then its Fourier components will have practically no correlation, except for the one required by the reality condition. The latter is defined by the twopoint correlator, specified by the spectrum $\mathcal{P}_{\zeta}(k)$ where $k$ is the wave number. On cosmological scales, the observed $\mathrm{CMB}$ anisotropy gives an almost scale-independent value $\mathcal{P}_{\zeta} \simeq\left(5 \times 10^{-5}\right)^{2}$.

According to typical scenarios for the generation of $\zeta$, the principle signal for non-Gaussianity would be the three-point correlator, specified by the nonlinearity parameter $f_{\mathrm{NL}}$. At present there is only an upper bound $\left|f_{\mathrm{NL}}\right| \lesssim 100$. Over the next few years, the bound will go down to $\left|f_{\mathrm{NL}}\right| \lesssim 5$ or so if there is no detection.

During inflation, the vacuum fluctuation of each canonically normalized light scalar field becomes a classical perturbation, with a nearly scale-independent and Gaussian spectrum. It is usually supposed that one or more of these perturbations is responsible for the primordial curvature perturbation $\zeta$. In this case, $\zeta$ is statistically homogeneous and isotropic, which means that its correlators are invariable under translations and rotations. Then $\mathcal{P}_{\zeta}(k)$ depends only on the magnitude of a momentum (wave vector) $\mathbf{k}$, and $f_{\mathrm{NL}}\left(k_{1}, k_{2}, k_{3}\right)$ depends on the lengths of the sides of a triangle.

Most of the proposals for generating $\zeta$ from the scalar field perturbations belong to one of two broad classes, which are distinguished by their prediction for $f_{\mathrm{NL}}$. If $\zeta$ is generated during single-field inflation one generally has $\left|f_{\mathrm{NL}}\right| \lesssim 10^{-2}$. More strongly, this bound applies to all single-field models in the squeezed configuration $k_{1} \simeq$ $k_{2} \gg k_{3}$. If instead $\zeta$ is generated at or after the end of inflation, $f_{\mathrm{NL}}$ becomes almost scale independent. In this case the prediction for $\left|f_{\mathrm{NL}}\right|$ is usually at least of order 1 and can be as big or bigger than the observational bound.

\footnotetext{
*m.karciauskas@lancaster.ac.uk

tk.dimopoulos1@lancaster.ac.uk

d.lyth@lancaster.ac.uk
}

Under the assumption of statistical isotropy, the constraints on $f_{\mathrm{NL}}$ from current observation at the $t w o-\sigma$ level are [1]

$$
-9<f_{\mathrm{NL}}^{\text {local }}<111, \quad-151<f_{\mathrm{NL}}^{\text {equil }}<253,
$$

where the label "local" can be taken to mean the squeezed configuration $k_{1} \simeq k_{2} \ll k_{3}$ and the label "equil" can be taken to mean the equilateral configuration $k_{1}=k_{2}=k_{3}$. The first result might be regarded as weak evidence for a $f_{\mathrm{NL}}^{\text {local }} \gg 1$, which if confirmed would rule out the generation of $\zeta$ during single-field inflation.

Recently, it has been suggested that the perturbation of some vector field may generate part or even all of the curvature perturbation. Such a thing is possible because the vacuum fluctuation of a vector field can generate an almost scale-independent and Gaussian spectrum although the condition for that to happen is rather special (e.g. it does not happen for a canonically normalized light vector field.) The contribution to $\zeta$ from a vector field perturbation is statistically homogeneous but not in general statistically isotropic. Then the spectrum may depend on the direction of $\mathbf{k}$, and the bispectrum may depend on the orientation of the triangle of vectors $\left(k_{1}, k_{2}, k_{3}\right)$.

Observational bounds on statistical anisotropy have not received much attention, and are not mentioned in the otherwise comprehensive analysis by the WMAP team [1]. As far as we know, the only available result [2] concerns the spectrum. It is parametrized in the form

$$
\mathcal{P}_{\zeta}(\mathbf{k})=\left[1+g(\hat{\mathbf{d}} \cdot \hat{\mathbf{k}})^{2}\right] \mathcal{P}_{\zeta}^{\text {iso }}(k),
$$

where $k=|\mathbf{k}|$ and the hats denote unit vectors. After taking account of all possible uncertainties, the conclusion from this study is $g \leq 0.31$ or so. We will adopt a bound $g \lesssim 0.1$ for definiteness.

Using the $\delta N$ formalism [3-6], one can write general formulas for the contribution of a vector field perturbation to the spectrum and the bispectrum. They were evaluated in Ref. [7] for the case that $\zeta$ is generated at the end of inflation, and in Ref. [8] for the case that $\zeta$ is generated by the curvaton mechanism. (Vector field inflation was also considered in Ref. [8] but we shall not consider it here.) It was found that the contribution to $\mathcal{P}_{\zeta}$ spectrum is of the 
form shown in Eq. (2). An analogous form for the contribution to $f_{\mathrm{NL}}$ is to be obtained in this work. These are the tree-level contributions. The one-loop contribution to $\mathcal{P}_{\zeta}$ is given in Ref. [8] but the one-loop contribution to $f_{\mathrm{NL}}$ is not known at the time of writing.

From the formula for $\mathcal{P}_{\zeta}$, one sees that its statistical anisotropy could easily be as big or bigger than the observational bound. The purpose of this paper is to consider also $f_{\mathrm{NL}}$. We want to know if a vector field contribution to $f_{\mathrm{NL}}$ could be big enough to observe, bearing in mind that its contribution to $\mathcal{P}_{\zeta}$ should respect the observational bound. We answer this question in the affirmative, and give explicit formulas for the dependence of $f_{\mathrm{NL}}$ on the orientation of the triangle.

\section{II. $f_{\text {NL }}$ INCLUDING VECTOR PERTURBATIONS}

The evolution of the curvature perturbation $\zeta$ on superhorizon scales is most readily described using a separate universe approach [3-6]. In a recent paper [8] the formalism was extended to take into account the possible statistical anisotropy in $\zeta$, where it was shown that once one includes perturbations of the vector field, the resulting curvature perturbation can be calculated up to quadratic terms using the following equation:

$$
\begin{aligned}
\zeta(\mathbf{x})= & N_{\phi} \delta \phi+N_{i}^{A} \delta A_{i}+\frac{1}{2} N_{\phi \phi}(\delta \phi)^{2}+\frac{1}{2} N_{\phi i}^{A} \delta \phi \delta A_{i} \\
& +\frac{1}{2} N_{i j}^{A} \delta A_{i} \delta A_{j},
\end{aligned}
$$

where $N$ is the number of e-folds of expansion of the unperturbed universe, the lower case roman letters denote spatial indices, and Einstein summation over those indices is assumed. The derivatives of $N$ with respect to the fields are denoted as

$$
\begin{gathered}
N_{\phi} \equiv \frac{\partial N}{\partial \phi}, \quad N_{i}^{A} \equiv \frac{\partial N}{\partial A_{i}}, \quad N_{\phi \phi} \equiv \frac{\partial^{2} N}{\partial \phi^{2}}, \\
N_{\phi i}^{A} \equiv \frac{\partial^{2} N}{\partial \phi \partial A_{i}}, \quad \text { and } \quad N_{i j}^{A} \equiv \frac{\partial^{2} N}{\partial A_{i} \partial A_{j}} .
\end{gathered}
$$

To obtain Eq. (3) it was assumed that the anisotropy of the expansion of the Universe is negligible. Even for the vector fields having a considerable contribution to the curvature perturbation the isotropic expansion of the Universe can be achieved at least in several ways, for example, with the oscillating massive vector field [9-11], using a triad of orthogonal vectors [12], a large number of identical randomly oriented vector fields [13], or if the contribution of the vector field(s) to the total energy density is negligible [7]. However, in general perturbations generated by vector fields will induce statistical anisotropy in $\zeta$. In Eq. (3) we assume only a single scalar field $\phi$ and a single vector field $A_{\mu}$.

We define the power spectrum and the bispectrum through the Fourier modes of $\zeta$ as

$$
\begin{aligned}
&\left\langle\zeta\left(\mathbf{k}_{1}\right), \zeta\left(\mathbf{k}_{2}\right)\right\rangle \equiv(2 \pi)^{3} \delta^{(3)}\left(\mathbf{k}_{1}+\mathbf{k}_{2}\right) \frac{2 \pi^{2}}{k^{3}} \mathcal{P}_{\zeta}\left(\mathbf{k}_{1}\right), \\
&\left\langle\zeta\left(\mathbf{k}_{1}\right) \zeta\left(\mathbf{k}_{2}\right) \zeta\left(\mathbf{k}_{3}\right)\right\rangle \equiv(2 \pi)^{3} \delta^{(3)}\left(\mathbf{k}_{1}+\mathbf{k}_{2}\right. \\
&\left.+\mathbf{k}_{3}\right) B_{\zeta}\left(\mathbf{k}_{1}, \mathbf{k}_{2}, \mathbf{k}_{3}\right),
\end{aligned}
$$

where normalization of Fourier components is chosen to be such that

$$
\zeta(\mathbf{k}) \equiv \int \zeta(\mathbf{x}) \mathrm{e}^{-i \mathbf{k} \cdot \mathbf{x}} d \mathbf{x} .
$$

Note that the power spectrum and the bispectrum are dependent on the direction of $\mathbf{k}$. The bispectrum $B_{\zeta}\left(\mathbf{k}_{1}, \mathbf{k}_{2}, \mathbf{k}_{3}\right)$ can be further separated into three parts: one due to perturbations in the scalar field, another part due to the vector field and a mixed term:

$$
\begin{aligned}
B_{\phi}\left(\mathbf{k}_{1}, \mathbf{k}_{2}, \mathbf{k}_{3}\right) \equiv & N_{\phi}^{2} N_{\phi \phi}\left[\frac{4 \pi^{4}}{k_{1}^{3} k_{2}^{3}} \mathcal{P}_{\phi}\left(k_{1}\right) \mathcal{P}_{\phi}\left(k_{2}\right)+\text { c.p. }\right] \\
B_{\phi A}\left(\mathbf{k}_{1}, \mathbf{k}_{2}, \mathbf{k}_{3}\right) \equiv & -\frac{1}{2} N_{\phi} N_{\phi, i}^{A}\left[\frac{4 \pi^{4}}{k_{1}^{3} k_{2}^{3}} \mathcal{P}_{\phi}\left(k_{1}\right) \mathcal{M}_{i}\left(\mathbf{k}_{2}\right)\right. \\
& +5 \text { perm }] \\
B_{A}\left(\mathbf{k}_{1}, \mathbf{k}_{2}, \mathbf{k}_{3}\right) \equiv & \frac{4 \pi^{4}}{k_{1}^{3} k_{2}^{3}} \mathcal{M}_{i}\left(\mathbf{k}_{1}\right) N_{i j}^{A} \mathcal{M}_{j}\left(\mathbf{k}_{2}\right)+\text { c.p., }
\end{aligned}
$$

where "c.p." stands for "cyclic permutations" and $k_{1}, k_{2}$, $k_{3}$ are the moduli of the vectors $\mathbf{k}_{1}, \mathbf{k}_{2}$, and $\mathbf{k}_{3}$.

The power spectrum $\mathcal{P}_{\phi}(k)$ in the above equations depends only on the modulus of $\mathbf{k}$ because we assumed that the expansion during inflation is isotropic. The vector $\mathcal{M}_{i}(\mathbf{k})$ characterizes perturbations of the vector field:

$$
\mathbf{M}(\mathbf{k}) \equiv \mathcal{P}_{+}(k) N_{A}\left[\hat{\mathbf{N}}^{A}+p(k) \hat{\mathbf{k}}\left(\hat{\mathbf{k}} \cdot \hat{\mathbf{N}}^{A}\right)+i q(k) \hat{\mathbf{k}} \times \hat{\mathbf{N}}^{A}\right]
$$

In this expression $N_{A}$ is the modulus of the vector $\mathbf{N}^{A}, \hat{\mathbf{N}}^{A}$ and $\hat{\mathbf{k}}$ are unit vectors defined by $\hat{\mathbf{N}}^{A} \equiv \mathbf{N}^{A} / N_{A}$ and $\hat{\mathbf{k}}=$ $\mathbf{k} / k$. The power spectrum for the longitudinal component is denoted by $\mathcal{P}_{0}(k)$ while $\mathcal{P}_{+}(k)$ and $\mathcal{P}_{-}(k)$ are the parity conserving and violating power spectra defined by

$$
\mathcal{P}_{ \pm} \equiv \frac{1}{2}\left(\mathcal{P}_{R} \pm \mathcal{P}_{L}\right)
$$

with $\mathcal{P}_{R}(k)$ and $\mathcal{P}_{L}(k)$ denoting the power spectra for the transverse components with right-handed and left-handed polarizations. Also we have defined $p(k)$ and $q(k)$ as

$$
p \equiv \frac{\mathcal{P}_{0}-\mathcal{P}_{+}}{\mathcal{P}_{+}} \quad \text { and } \quad q \equiv \frac{\mathcal{P}_{-}}{\mathcal{P}_{+}} .
$$

Because of the isotropic expansion during inflation the power spectra $\mathcal{P}_{0}$ and $\mathcal{P}_{ \pm}$depend only on the modulus of $\mathbf{k}$.

The curvature perturbation power spectrum $\mathcal{P}_{\zeta}(\mathbf{k})$ may be separated into isotropic and anisotropic parts [14]: 


$$
\mathcal{P}_{\zeta}(\mathbf{k})=\mathcal{P}_{\zeta}^{\text {iso }}(k)\left[1+g(k)\left(\hat{\mathbf{N}}^{A} \cdot \hat{\mathbf{k}}\right)^{2}\right]
$$

where the amount of anisotropy at each scale is parametrized by

$$
g \equiv N_{A}^{2} \frac{\mathcal{P}_{0}-\mathcal{P}_{+}}{\mathcal{P}_{\zeta}^{\text {iso }}}
$$

The isotropic part of the spectrum is

$$
\mathcal{P}_{\zeta}^{\text {iso }} \equiv N_{\phi}^{2} \mathcal{P}_{\phi}+N_{A}^{2} \mathcal{P}_{+}
$$

In this paper we will calculate $f_{\mathrm{NL}}$ for both configurations: equilateral, in which $k_{1}=k_{2}=k_{3}$, and squeezed, in which $k_{1} \simeq k_{2} \gg k_{3}$. In the equilateral configuration the bispectra from Eqs. (8) become

$$
\begin{aligned}
\mathcal{B}_{\phi}^{\text {equil }}\left(\mathbf{k}_{1}, \mathbf{k}_{2}, \mathbf{k}_{3}\right)= & 3 N_{\phi}^{2} N_{\phi \phi} \mathcal{P}_{\phi}^{2}\left(k_{1}\right), \\
\mathcal{B}_{A \phi}^{\text {equil }}\left(\mathbf{k}_{1}, \mathbf{k}_{2}, \mathbf{k}_{3}\right)= & -N_{\phi} N_{\phi, i}^{A} \mathcal{P}_{\phi}\left(k_{1}\right) \\
& \times\left[\mathcal{M}_{i}\left(\mathbf{k}_{1}\right)+\mathcal{M}_{i}\left(\mathbf{k}_{2}\right)+\mathcal{M}_{i}\left(\mathbf{k}_{3}\right)\right], \\
\mathcal{B}_{A}^{\text {equil }}\left(\mathbf{k}_{1}, \mathbf{k}_{2}, \mathbf{k}_{3}\right)= & \mathcal{M}_{i}\left(\mathbf{k}_{1}\right) N_{i j}^{A} \mathcal{M}_{j}\left(\mathbf{k}_{2}\right)+\text { c.p. },
\end{aligned}
$$

where we have defined for the equilateral configuration

$$
\mathcal{B}_{\zeta}^{\text {equil }}\left(\mathbf{k}_{1}, \mathbf{k}_{2}, \mathbf{k}_{3}\right) \equiv\left(\frac{k_{1}^{3}}{2 \pi^{2}}\right)^{2} B_{\zeta}^{\text {equil }}\left(\mathbf{k}_{1}, \mathbf{k}_{2}, \mathbf{k}_{3}\right),
$$

and $\mathcal{B}_{\zeta}^{\text {equil }}=\mathcal{B}_{\phi}^{\text {equil }}+\mathcal{B}_{A \phi}^{\text {equil }}+\mathcal{B}_{A}^{\text {equil }}$. In this case the nonlinearity parameter $f_{\mathrm{NL}}^{\text {equil }}$ is expressed through the power spectrum and the bispectrum as

$$
\frac{6}{5} f_{\mathrm{NL}}^{\text {equil }}=\frac{\mathcal{B}_{\zeta}^{\text {equil }}\left(\mathbf{k}_{1}, \mathbf{k}_{2}, \mathbf{k}_{3}\right)}{3 \mathcal{P}_{\zeta}^{\text {iso }}(k)^{2}} .
$$

Observations give a limit on the anisotropy $g \lesssim 0.1$ [2]. Therefore, since the anisotropic contribution to the curvature perturbation is subdominant compared to the isotropic one, we have included only $\mathcal{P}_{\zeta}^{\text {iso }}$ into the above expression of $f_{\mathrm{NL}}^{\text {equil }}$.

For the squeezed configuration two of the vectors have almost identical lengths but opposite directions, $\mathbf{k}_{1} \simeq$ $-\mathbf{k}_{2}$, but the third vector $\mathbf{k}_{3}$ is of much smaller modulus than the other two and almost perpendicular to them. For this configuration Eqs. (8) take the form

$$
\begin{aligned}
\mathcal{B}_{\phi}^{\text {local }}\left(\mathbf{k}_{1}, \mathbf{k}_{2}, \mathbf{k}_{3}\right)= & 2 N_{\phi}^{2} N_{\phi \phi} \mathcal{P}_{\phi}\left(k_{1}\right) \mathcal{P}_{\phi}\left(k_{3}\right), \\
\mathcal{B}_{A \phi}^{\text {local }}\left(\mathbf{k}_{1}, \mathbf{k}_{2}, \mathbf{k}_{3}\right)= & -N_{\phi} N_{\phi, i}^{A}\left\{\mathcal{P}_{\phi}\left(k_{1}\right) \mathcal{M}_{i}\left(\mathbf{k}_{3}\right)\right. \\
& \left.+\mathcal{P}_{\phi}\left(k_{3}\right) \operatorname{Re}\left[\mathcal{M}_{i}\left(\mathbf{k}_{1}\right)\right]\right\} \\
\mathcal{B}_{A}^{\text {local }}\left(\mathbf{k}_{1}, \mathbf{k}_{2}, \mathbf{k}_{3}\right)= & 2 \operatorname{Re}\left[\mathcal{M}_{i}\left(\mathbf{k}_{1}\right)\right] N_{i j}^{A} \operatorname{Re}\left[\mathcal{M}_{j}\left(\mathbf{k}_{3}\right)\right],
\end{aligned}
$$

where $\operatorname{Re}[\ldots]$ means the real part and $\mathcal{B}_{\zeta}^{\text {local }}\left(\mathbf{k}_{1}, \mathbf{k}_{2}, \mathbf{k}_{3}\right)$ is defined similarly to Eq. (16)

$$
\mathcal{B}_{\zeta}^{\text {local }}\left(\mathbf{k}_{1}, \mathbf{k}_{2}, \mathbf{k}_{3}\right) \equiv \frac{k_{1}^{3} k_{3}^{3}}{4 \pi^{4}} B_{\zeta}^{\text {local }}\left(\mathbf{k}_{1}, \mathbf{k}_{2}, \mathbf{k}_{3}\right) .
$$

Then, the nonlinearity parameter $f_{\mathrm{NL}}^{\text {local }}$ in the squeezed configuration becomes

$$
\frac{6}{5} f_{\mathrm{NL}}^{\text {local }}=\frac{\mathcal{B}_{\zeta}^{\text {local }}\left(\mathbf{k}_{1}, \mathbf{k}_{2}, \mathbf{k}_{3}\right)}{2 \mathcal{P}_{\zeta}^{\text {iso }}\left(k_{1}\right) \mathcal{P}_{\zeta}^{\text {iso }}\left(k_{3}\right)} .
$$

Having defined our notation, in the following sections we will consider two particular examples. But before that, we wish to point out an important subtlety concerning the vector field $A_{\mu}$. By $A_{i}$ we refer to the spatial components of the physical vector field $A_{i}=B_{i} / a$, where $a$ is the scale factor in the Friedmann-Robertson-Walker (FRW) universe. The field $B_{i}$, which enters the Lagrangian, is the comoving vector field with the expansion of the universe factored out. In FRW spacetime the temporal components of the physical and comoving fields are the same, $A_{0}=B_{0}$ $[9,10]$.

\section{ANISOTROPY IN THE VECTOR CURVATON MODEL}

In this section we study the non-Gaussianity in the curvature perturbation for a model of slow roll inflation with an additional $U(1)$ vector field which decays some time after reheating and contributes to the total curvature perturbation following the curvaton mechanism [15]. For this contribution to be non-negligible, the vector field must undergo particle production during inflation and obtain a superhorizon spectrum of perturbations. But the massless $U(1)$ field is conformally invariant and, consequently, its quantum fluctuations are not amplified during inflation. This means that in order for such a vector field to undergo particle production we have to brake its conformality [16]. Another problem is that a dominant homogeneous (homogenized by inflation) vector field could make the expansion of the Universe strongly anisotropic, which is in contradiction with observations.

One way out of this problem can be the curvaton mechanism $[15,17,18]$. The usual curvaton scenario incorporates two scalar fields: one that drives inflation and another one, called curvaton, which produces the curvature perturbation. During inflation the curvaton is subdominant. However, after reheating the universe is radiation dominated and its energy density is diluted as $\rho_{r} \propto a^{-4}$. If the energy density of the curvaton field decreases slower than $a^{-4}$, at some moment it can dominate (or nearly dominate) the Universe and impose its own curvature perturbation. This is the basic idea of the curvaton mechanism.

Here we consider a massive vector field acting as the curvaton. Before dominating the vector curvaton field is rapidly oscillating in a quasiharmonic manner. As shown in Ref. [9], the oscillating vector field behaves as a pressureless isotropic fluid and can dominate without generating a large-scale anisotropy. 


\section{A. The generic treatment}

Here we obtain analytic expressions for the nonlinearity parameter $f_{\mathrm{NL}}$ without assuming a specific vector curvaton model. In contrast to the original curvaton idea we include as well perturbations generated during inflation by the light scalar field (for a similar study in the scalar curvaton case see Refs. [19,20].

Some time after reheating the mass of the vector field becomes bigger than the Hubble parameter, the field starts to oscillate. In Ref. [8] it was shown that for the oscillating vector field $N_{i}^{A}$ and $N_{i j}^{A}$ are equal to

$$
\begin{aligned}
& N_{i}^{A}=\frac{2}{3} r \frac{A_{i}}{A^{2}}, \\
& N_{i j}^{A}=\frac{2}{3} r \frac{\delta_{i j}}{A^{2}},
\end{aligned}
$$

where $A \equiv|\mathbf{A}|$ is evaluated just before the vector field decays and the parameter $r$ is defined as

$$
r \equiv \frac{3 \rho_{A}}{3 \rho_{A}+4 \rho_{r}}=\frac{3 \Omega_{A}}{4-\Omega_{A}}
$$

with $\rho_{A}$ being the energy density of the vector field just before its decay (taken to be sudden), $\Omega_{A} \equiv \rho_{A} / \rho$ and $\rho=$ $\rho_{A}+\rho_{r}$. Using Eq. (21) the isotropic part of the total power spectrum in Eq. (14) becomes

$$
\mathcal{P}_{\zeta}^{\text {iso }}=N_{\phi}^{2} \mathcal{P}_{\phi}\left(1+\beta \frac{\mathcal{P}_{+}}{\mathcal{P}_{\phi}}\right)
$$

where we defined

$$
\beta \equiv\left(\frac{N_{A}}{N_{\phi}}\right)^{2} .
$$

Then the vector part of the bispectrum for equilateral configuration in Eq. (15) reduces to

$$
\begin{aligned}
\mathcal{B}_{A}^{\text {equil }}\left(\mathbf{k}_{1}, \mathbf{k}_{2}, \mathbf{k}_{3}\right) & \\
= & \left(\frac{2}{3} \frac{r}{A}\right)^{3} \frac{1}{A} \mathcal{P}_{+}\left(k_{1}\right) \mathcal{P}_{+}\left(k_{2}\right)\left\{1+p\left(k_{1}\right) A_{1}^{2}+p\left(k_{2}\right) A_{2}^{2}\right. \\
& +A_{1} A_{2}\left[q\left(k_{1}\right) q\left(k_{2}\right)-\frac{1}{2} p\left(k_{1}\right) p\left(k_{2}\right)\right] \\
& +i \sqrt{\frac{3}{4}-\left(A_{1}^{2}+A_{1} A_{2}+A_{2}^{2}\right)\left[A_{1} p\left(k_{1}\right) q\left(k_{2}\right)\right.} \\
& \left.\left.-A_{2} p\left(k_{2}\right) q\left(k_{1}\right)\right]+\frac{1}{2} q\left(k_{1}\right) q\left(k_{2}\right)\right\}+ \text { c.p. }
\end{aligned}
$$

In the above we used the notation $A_{1} \equiv \hat{\mathbf{A}} \cdot \hat{\mathbf{k}}_{1}$, etc., where $\hat{\mathbf{A}}=\mathbf{A} / A$. Because the configuration of wave vectors $\hat{\mathbf{k}}_{1}$, $\hat{\mathbf{k}}_{2}$, and $\hat{\mathbf{k}}_{3}$ is equilateral, with the angle between any two of them being $2 \pi / 3$, we find $\hat{\mathbf{k}}_{1} \cdot \hat{\mathbf{k}}_{2}=\hat{\mathbf{k}}_{1} \cdot \hat{\mathbf{k}}_{3}=\hat{\mathbf{k}}_{2} \cdot \hat{\mathbf{k}}_{3}=$ $-\frac{1}{2}$. Equation (26) simplifies further if we consider a scale invariant power spectrum and the expression for $f_{\mathrm{NL}}^{\text {equil }}$ becomes

$$
\frac{6}{5} f_{\mathrm{NL}}^{\text {equil }}=\beta^{2} \mathcal{P}_{+}^{2} \frac{3}{2 r} \frac{\left(1+\frac{1}{2} q^{2}\right)+\left[p+\frac{1}{8}\left(p^{2}-2 q^{2}\right)\right] A_{\perp}^{2}}{\left(\mathcal{P}_{\phi}+\beta \mathcal{P}_{+}\right)^{2}},
$$

where we have taken into account that the non-Gaussianity generated during the single-field inflation is negligible. The quantity $A_{\perp} \leq 1$ is the modulus of the projection of the unit vector $\hat{\mathbf{A}}$ onto the plane containing the three vectors $\hat{\mathbf{k}}_{1}, \hat{\mathbf{k}}_{2}$, and $\hat{\mathbf{k}}_{3}$. The calculation of $A_{\perp}$ in the equilateral configuration is explained in more detail in the appendix.

For the squeezed configuration the bispectrum from the vector field perturbation in Eqs. (18) becomes

$$
\begin{aligned}
\mathcal{B}_{A}^{\text {local }}\left(\mathbf{k}_{1}, \mathbf{k}_{2}, \mathbf{k}_{3}\right)= & 2\left(\frac{2}{3} \frac{r}{A}\right)^{3} \frac{1}{A} \mathcal{P}_{+}\left(k_{1}\right) \mathcal{P}_{+}\left(k_{3}\right) \\
& \times\left[1+p\left(k_{1}\right) A_{1}^{2}+p\left(k_{3}\right) A_{3}^{2}\right] .
\end{aligned}
$$

Working as in the equilateral case, we find that the nonlinearity parameter for the scale invariant power spectra is

$$
\frac{6}{5} f_{\mathrm{NL}}^{\text {local }}=\beta^{2} \mathcal{P}_{+}^{2} \frac{3}{2 r} \frac{1+p A_{\perp}^{2}}{\left(\mathcal{P}_{\phi}+\beta \mathcal{P}_{+}\right)^{2}} .
$$

As one can see from the above equations, $f_{\mathrm{NL}}$ is, in general, dependent on $A_{\perp}$, in both configurations. This means that $f_{\mathrm{NL}}$ is anisotropic and that the amount of non-Gaussianity is correlated with the statistical anisotropy. However, from Eqs. (10), (11), and (13) it is clear that, if particle production is isotropic (i.e. $\mathcal{P}_{0}=\mathcal{P}_{+}$and $\left.\mathcal{P}_{-}=0\right)$ then $p=q=0$ and the above expressions for $f_{\mathrm{NL}}^{\text {equil }}$ and $f_{\mathrm{NL}}^{\text {local }}$ become isotropic too and both reduce to $f_{\mathrm{NL}}=5 / 4 r$ as in the scalar curvaton scenario, where we have assumed that $\mathcal{P}_{\phi} \ll \mathcal{P}_{+}$, i.e. that the dominant contribution to the curvature perturbation is due to the vector curvaton field only.

\section{B. $f_{\mathrm{NL}}$ for nonminimally coupled vector curvaton}

In Ref. [9] it was shown that a vector field can attain a scale invariant perturbation spectrum if its mass during inflation is equal to $m^{2}=-2 H^{2}$. One way to achieve a negative mass squared of this magnitude is to introduce a nonminimal coupling of the vector field to gravity of the form $\frac{1}{6} R B^{\mu} B_{\mu}$, where $R$ is the Ricci scalar. The idea of such a nonminimally coupled vector curvaton was introduced in Ref. [11]. In that paper and in Ref. [8] it was shown that the power spectra for different polarizations are

$$
\mathcal{P}_{+}=\left(\frac{H}{2 \pi}\right)^{2}, \quad \mathcal{P}_{-}=0, \quad \text { and } \quad \mathcal{P}_{0}=2\left(\frac{H}{2 \pi}\right)^{2} .
$$

One notices that the parity conserving transverse power spectrum and the power spectrum generated during the single scalar field inflation are equal, i.e. $\mathcal{P}_{+}=\mathcal{P}_{\phi}$. Thus the isotropic part of the curvature perturbation spec- 
trum can be written as

$$
\mathcal{P}_{\zeta}^{\text {iso }}=\mathcal{P}_{\phi} N_{\phi}^{2}(1+\beta),
$$

while the anisotropy parameter from Eq. (13) becomes

$$
g=\frac{\beta}{1+\beta} .
$$

Using Eq. (30) we find

$$
p=1 \quad \text { and } \quad q=0 .
$$

Thus, the anisotropy in the vector field is rather strong, which means that it will have to remain subdominant, i.e. $\Omega_{A} \ll 1$. Using this and Eq. (27), the $f_{\mathrm{NL}}^{\text {equil }}$ for the nonminimally coupled vector curvaton is found to be

$$
\frac{6}{5} f_{\mathrm{NL}}^{\text {equil }}=2 \frac{\beta^{2}}{\Omega_{A}}\left(1+\frac{9}{8} A_{\perp}^{2}\right) .
$$

Similarly, $f_{\mathrm{NL}}^{\text {local }}$ for the squeezed configuration in Eq. (29) is

$$
\frac{6}{5} f_{\mathrm{NL}}^{\text {local }}=2 \frac{\beta^{2}}{\Omega_{A}}\left(1+A_{\perp}^{2}\right) .
$$

Since $\mathcal{P}_{+}=\frac{1}{2} \mathcal{P}_{0}=\mathcal{P}_{\phi}=\left(\frac{H}{2 \pi}\right)^{2}$, for the typical values of the perturbations we have $\delta \phi \sim \delta A_{i} \sim H$. This means that, in order for the vector field contribution to be subdominant, we require $N_{A} \ll N_{\phi}$ (c.f. Eq. (3)). Hence, $\beta \ll$ 1 and $g \simeq \beta$. Thus, in view of Eqs. (34) and (35), we see that $f_{\mathrm{NL}} \sim g^{2} / \Omega_{A}$. Therefore, we find that the nonGaussianity is determined by the magnitude of the statistical anisotropy.

This prediction is valid in the regime $|\delta A / A| \ll 1$ which corresponds to $\Omega_{A}^{2} \gtrsim \mathcal{P}_{\zeta} \beta$, which implies $f_{\mathrm{NL}} \lesssim$ $g^{3 / 2} / \sqrt{\mathcal{P}_{\zeta}}$. For smaller $\Omega_{A}$, the contribution of the vector field perturbation to $\zeta$ is of order $\Omega_{A}\left[\delta A /\left(\overline{\delta A^{2}}\right)^{1 / 2}\right]$. In other words, it is of order $\Omega_{A}$ and is the square of a Gaussian quantity. The resulting prediction for its contribution to $f_{\mathrm{NL}}$ would be given by a one-loop formula which has not been evaluated at the time of writing.

\section{ANISOTROPY GENERATED AT THE END OF INFLATION}

As another example, we consider the generation of an anisotropic power spectrum at the end of inflation. The idea is based on Ref. [21] where it was shown that in hybrid inflation models the generation of curvature perturbations can be realized due to inhomogeneous end of inflation. Yokoyama and Soda [7] used this idea to generate the anisotropic contribution to the total curvature perturbations. In their model the anisotropy is generated at the end of inflation due to the vector field coupling with the waterfall field. In this section we calculate the non-
Gaussianity of the model in Ref. [7] using our notation in Sec. II.

In this model there are two components of the curvature perturbation: one generated during inflation and an anisotropic one, generated by a vector field at the end of inflation:

$$
\zeta=\zeta_{\text {inf }}+\zeta_{\text {end }}
$$

The first component is due to the perturbation generated during inflation. The second component is due to the perturbation of the vector field. Reference [7] considers a massless, $U(1)$ vector field. Without parity violating terms the power spectra for left-handed and right-handed polarizations are equal, while the longitudinal polarization is absent for a massless field. In this situation we find that parameters $p(k)$ and $q(k)$ from Eq. (11) become

$$
p=-1 \text { and } q=0 \text {. }
$$

The conformal invariance of the $U(1)$ vector field is broken through a noncanonical kinetic function of the form $f^{2}(t) F_{\mu \nu} F^{\mu \nu}$, where $F_{\mu \nu}=\partial_{\mu} B_{\nu}-\partial_{\nu} B_{\mu}$ is the field strength tensor and $B_{\mu}$-comoving vector field. This form of conformal invariance braking was considered in many papers (e.g. Refs. [10,22-25]) where it was found that a scale invariant perturbation spectrum is obtained if $f \propto a$ :

$$
\mathcal{P}_{+}=\left(\frac{H}{2 \pi f}\right)^{2}=\mathcal{P}_{\phi} f^{-2} .
$$

So the isotropic part in Eq. (14) of the power spectrum becomes

$$
\mathcal{P}_{\zeta}^{\text {iso }}=\mathcal{P}_{\phi} N_{\phi}^{2}(1+\beta) .
$$

This is of the same form as with the vector curvaton model but with different $\beta$ :

$$
\beta=\left(\frac{N_{A}}{N_{\phi} f}\right)^{2} .
$$

The anisotropy parameter in Eq. (13) in this model becomes

$$
g=-\frac{\beta}{1+\beta} .
$$

Taking into account Eq. (37), the vector $\mathcal{M}_{i}(\mathbf{k})$ in Eq. (9) reduces to the simple form

$$
\mathcal{M}(\mathbf{k})=N_{A} \mathcal{P}_{\phi} f^{-2}\left[\hat{\mathbf{N}}^{A}-\hat{\mathbf{k}}\left(\hat{\mathbf{N}}^{A} \cdot \hat{\mathbf{k}}\right)\right] .
$$

To calculate $f_{\mathrm{NL}}$ we consider a specific example of hybrid inflation with the potential

$$
\begin{aligned}
V\left(\phi, \chi, B^{\mu}\right)= & V_{0}+\frac{1}{2} m_{\phi}^{2} \phi^{2}-\frac{1}{2} m_{\chi}^{2} \chi^{2}+\frac{1}{4} \lambda \chi^{4} \\
& +\frac{1}{2} \lambda_{\phi} \phi^{2} \chi^{2}+\frac{1}{2} \lambda_{A} \chi^{2} B^{\mu} B_{\mu},
\end{aligned}
$$

where $\phi$ is the inflaton and $\chi$ is the waterfall field. The 
effective mass of the waterfall field for this potential is

$$
m_{\mathrm{eff}}^{2}=-m_{\chi}^{2}+\lambda_{\phi} \phi^{2}-\lambda_{A} A_{i} A_{i},
$$

where Einstein summation is assumed and we used $A_{i} \equiv$ $B_{i} / a$ and the Coulomb gauge in which $A_{0}=0$ and $\partial_{i} A^{i}=$ 0 . Inflation ends when the inflaton reaches a critical value $\phi_{c}$ where the effective mass of the waterfall field becomes tachyonic. But one can see from Eq. (44) that the critical value is a function of the vector field $\phi_{c}=\phi_{c}(A)$. With this in mind the vectors $N_{i}^{A}$ and $N_{i j}^{A}$ can be readily calculated:

$$
N_{i}^{A}=\frac{\partial N}{\partial \phi_{c}} \frac{\partial \phi_{c}}{\partial A_{i}}=N_{c} \frac{\lambda_{A}}{\lambda_{\phi}} \frac{A_{i}}{\phi_{c}},
$$

and

$$
\begin{aligned}
N_{i j}^{A} & =\frac{\partial N}{\partial \phi_{c}} \frac{\partial^{2} \phi_{c}}{\partial A^{i} \partial A^{j}}+\frac{\partial^{2} N}{\partial \phi_{c}^{2}} \frac{\partial \phi_{c}}{\partial A_{i}} \frac{\partial \phi_{c}}{\partial A_{j}} \\
& =\frac{N_{A}^{2}}{\phi_{c} N_{c}}\left(C^{2} \delta_{i j}-\hat{A}_{i} \hat{A}_{j}\right),
\end{aligned}
$$

where we have defined

$$
N_{c}=\frac{\partial N}{\partial \phi_{c}} \quad \text { and } \quad C \equiv \sqrt{\frac{\lambda_{\phi}}{\lambda_{A}}} \frac{\phi_{c}}{A},
$$

where $A$ is evaluated at the end of inflation and we used the fact that $N_{c c} / N_{c}^{2} \sim N_{\phi \phi} / N_{\phi}^{2} \sim \mathcal{O}(\epsilon)$ under the slow roll approximation [21], where $\epsilon$ is the slow roll parameter defined as $\epsilon \equiv \frac{1}{2} M_{\mathrm{P}}^{2}\left(V^{\prime} / V\right)^{2}$, with the prime denoting derivatives with respect to the inflaton. As mentioned earlier the total of perturbations consists of two components: perturbations of the scalar and vector fields. This gives the following bispectrum in the equilateral configuration

$$
\begin{aligned}
\mathcal{B}_{\zeta}^{\text {equil }}\left(\mathbf{k}_{1}, \mathbf{k}_{2}, \mathbf{k}_{3}\right)= & \mathcal{B}_{\phi}^{\text {equil }}\left(\mathbf{k}_{1}, \mathbf{k}_{2}, \mathbf{k}_{3}\right)+\mathcal{B}_{A}^{\text {equil }}\left(\mathbf{k}_{1}, \mathbf{k}_{2}, \mathbf{k}_{3}\right) \\
= & 3 \mathcal{P}_{\phi}^{2} N_{\phi}^{2} N_{\phi \phi} \\
& +\left[\mathcal{M}_{i}\left(\mathbf{k}_{1}\right) N_{i j}^{A} \mathcal{M}_{j}\left(\mathbf{k}_{2}\right)+\text { c.p. }\right] \\
= & \mathcal{P}_{\phi}^{2} N_{\phi}^{4} \frac{\delta^{2}}{N_{c} \phi_{c}} 3\left[\left(C^{2}-1\right)\right. \\
& \left.-\left(\frac{7}{8} C^{2}-1\right) A_{\perp}^{2}-\frac{3}{16} A_{\perp}^{4}\right]
\end{aligned}
$$

The mixed term $\mathcal{B}_{\phi A}^{\text {equil }}$ is absent from Eq. (48) because in this model $N_{\phi i}^{A}=0$. By inserting Eq. (39) into (48) we obtain

$$
\frac{6}{5} f_{\mathrm{NL}}^{\text {equil }}=\eta g^{2}\left[\left(C^{2}-1\right)-\left(\frac{7}{8} C^{2}-1\right) A_{\perp}^{2}-\frac{3}{16} A_{\perp}^{4}\right],
$$

where the slow parameter $\eta$ is equal to $\eta=m_{\phi}^{2} M_{\mathrm{P}}^{2} / V_{0}$ and $M_{\mathrm{P}} N_{c}=1 / \sqrt{2 \epsilon_{c}}$, with $\epsilon_{c}$ being the slow roll parameter at the end of inflation. Similarly, for the squeezed configuration we find

$$
\frac{6}{5} f_{\mathrm{NL}}^{\text {local }}=\eta g^{2}\left[\left(C^{2}-1\right)\left(1-A_{\perp}^{2}\right)-\frac{1}{4}(\sin \varphi)^{2} A_{\perp}^{4}\right] .
$$

In this equation $\varphi$ is the angle between vectors $\mathbf{k}_{1}$ and $\mathbf{A}_{\perp}$.
Again, we find that $f_{\mathrm{NL}}^{\text {equil }}$ and $f_{\mathrm{NL}}^{\text {local }}$ are functions of $A_{\perp}$, i.e. they are anisotropic and correlated with the statistical anisotropy. Also the level of non-Gaussianity is proportional to the anisotropy parameter squared, $f_{\mathrm{NL}} \propto g^{2}$, as in the nonminimally coupled vector curvaton model.

\section{DISCUSSION}

In this paper we have calculated the amount of the nonGaussianity generated by the anisotropic part of the curvature perturbation. We have also considered two specific models to generate the anisotropic curvature perturbation from the vector fields and calculated the non-Gaussianity in detail for those models. The results were given for the equilateral and squeezed configurations in Eqs. (34) and (35) for the model of nonminimally coupled vector curvaton and in Eqs. (49) and (50) for the end-of-inflation scenario. We have shown that $f_{\mathrm{NL}}$ generated by the vector field is anisotropic and that it is correlated with the amount and direction of the statistical anisotropy.

Although in some specific models it would be possible to generate the statistically isotropic curvature perturbation only from the vector field, however, in general, perturbations generated by the vector field are anisotropic. From observations the bound on statistically anisotropic contribution to the total power spectrum is constrained to be less than about $10 \%$. In this case one can estimate the maximal $f_{\mathrm{NL}}$ caused by the statistically anisotropic curvature perturbation on fairly general grounds.

Let us assume that the non-Gaussianity is produced solely due to the vector field perturbations. If this is so then Eqs. (17) and (20) suggest $f_{\mathrm{NL}} \sim \mathcal{B}_{\zeta} / \mathcal{P}_{\zeta}^{2}$, where we consider that the anisotropic contribution to the curvature perturbation is subdominant, i.e. $\mathcal{P}_{\zeta} \simeq \mathcal{P}_{\zeta}^{\text {iso }}$. According to Eqs. (16) and (19) we have $\mathcal{B}_{\zeta} \sim \mathcal{M}^{2} N_{A A}$, where $\mathcal{M} \equiv$ $|\mathcal{M}|$ and $N_{A A} \equiv\left\|N_{i j}^{A}\right\|$. Thus, we have $f_{\mathrm{NL}} \sim$ $\mathcal{M}^{2} N_{A A} / \mathcal{P}_{\zeta}^{2}$.

Now, $\mathcal{M}$ depends on the mechanism which breaks the conformality of the vector field and is responsible for the generation of its superhorizon perturbation spectrum. If this mechanism does not introduce additional mass scales, then, on dimensional grounds, we expect the anisotropy in the vector field perturbation to be of order unity, i.e. $|p|$, $|q| \sim \mathcal{O}(1)$, barring cancellations such as due to parity invariance (which results in $q=0$ ) or an isotropic particle production, which gives $p=q=0$ and generates no statistical anisotropy. In our examples in Secs. III and IV we indeed analyzed such a situation, where the effective mass squared of the vector field during inflation $m^{2}=\frac{1}{6} R=$ $-2 H^{2}$ or the time dependence of the kinetic function $\dot{f} / f=H$ were both determined by the dynamics of the expansion and given by $H$, the only scale in the theory. If the anisotropy in the vector field perturbation is of order unity, then Eq. (9) gives $\mathcal{M} \sim \mathcal{P}_{A} N_{A}$, where $\mathcal{P}_{A}=$ $2 \mathcal{P}_{+}+\mathcal{P}_{0}$ is the power spectrum of the total vector field 
perturbation given by $\mathcal{P}_{A}=\frac{k^{3}}{2 \pi^{2}} \sum_{i}\left|\delta A_{i}\right|^{2}$ in the superhorizon limit. Putting the above together we obtain

$$
f_{\mathrm{NL}} \sim \frac{\mathcal{B}_{\zeta}}{\mathcal{P}_{\zeta}^{2}} \sim \frac{\mathcal{M}^{2} N_{A A}}{\mathcal{P}_{\zeta}^{2}} \sim \frac{\mathcal{P}_{A}^{2} N_{A}^{2} N_{A A}}{\mathcal{P}_{\zeta}^{2}} .
$$

Since we are working in the regime where $|\delta A / A| \ll 1$ we expect the higher order contribution to $\zeta$ in Eq. (3) from the vector field to be subdominant, i.e. $N_{A} \delta A>N_{A A} \delta A^{2}$. Considering that the typical value of the vector field perturbation is $\delta A \sim \sqrt{\mathcal{P}_{A}}$, we obtain the bound $f_{\mathrm{NL}}<$ $\left(\mathcal{P}_{A}^{1 / 2} N_{A}\right)^{3} / \mathcal{P}_{\zeta}^{2}$. As is evident from Eq. (3), the contribution of the vector field to $\zeta$ is given by $\zeta_{A} \sim \sqrt{\mathcal{P}_{\zeta_{A}}} \sim N_{A} \sqrt{\mathcal{P}_{A}}$, where $\mathcal{P}_{\zeta_{A}}$ is the power spectrum of the anisotropic curvature perturbation. With this in mind, the upper bound to $f_{\mathrm{NL}}$ becomes

$$
f_{\mathrm{NL}} \mathcal{P}_{\zeta}^{1 / 2}<\left(\frac{\mathcal{P}_{\zeta_{A}}}{\mathcal{P}_{\zeta}}\right)^{3 / 2}
$$

Because the vector field contribution to the total curvature perturbation must be subdominant, Eq. (12) suggests that the anisotropy of the curvature perturbation is $g \sim$ $\mathcal{P}_{\zeta_{A}} / \mathcal{P}_{\zeta}$. Using this and also that $\mathcal{P}_{\zeta} \approx 5 \times 10^{-5}$, we find that the maximum value of $f_{\mathrm{NL}}$ generated by the statistically anisotropic contribution to the curvature perturbation has to be

$$
f_{\mathrm{NL}}^{\max } \sim 10^{3}\left(\frac{g}{0.1}\right)^{3 / 2} .
$$

Our results in Eqs. (34), (35), (49), and (50) apply if $f_{\mathrm{NL}}$ is below this value.

\section{CONCLUSIONS}

It is clear that the study of vector field contributions to the primordial curvature perturbation is just beginning. Given a scale invariant and Gaussian vector field perturbation, the calculation of $\zeta$ from the $\delta N$ formalism is straightforward and should now be done for the full range of scenarios that have already been explored for the contribution of scalar field perturbations. Also, the Feynman graph formalism available for the scalar field case should be generalized to cover the vector field contributions. At a deeper level, one also wishes to understand how the scale invariant perturbation can be generated. One would also like a fuller understanding of the generation of perturbations from the vacuum fluctuation when the expansion of the unperturbed Universe is anisotropic, since that can easily happen in the presence of vector fields.

For the moment though, it is most urgent to confront specific predictions for the form of the anisotropy with observation. Specifically, we want to know what constraint is placed by observation on a contribution to $f_{\mathrm{NL}}$ of the form in Eqs. (34) and (35) or Eqs. (49) and (50). It seems quite possible that with the latter prediction, valid for the squeezed configuration, one might find a nonzero value at better than the 2- $\sigma$ level that is already found for the isotropic case. Such a finding, if confirmed, would be a smoking gun for a vector field contribution to the curvature perturbation.

\section{ACKNOWLEDGMENTS}

This work was supported (in part) by the European Union through the Marie Curie Research and Training Network "UniverseNet" (MRTN-CT-2006-035863) and by STFC (PPARC) Grant No. PP/D000394/1.

\section{APPENDIX: CALCULATION OF $A_{\perp}$ IN THE EQUILATERAL CONFIGURATION}

First note that in the equilateral configuration $\hat{\mathbf{k}}_{1}+$ $\hat{\mathbf{k}}_{2}=-\hat{\mathbf{k}}_{3}$. This gives $A_{1}+A_{2}=-A_{3}$ and

$$
A_{1}^{2}+A_{2}^{2}+A_{3}^{2}=2\left(A_{1}^{2}+A_{1} A_{2}+A_{2}^{2}\right) ;
$$

$A_{1} A_{2}+A_{2} A_{3}+A_{3} A_{1}=-\left(A_{1}^{2}+A_{1} A_{2}+A_{2}^{2}\right)$;

$A_{1}^{2} A_{2}^{2}+A_{2}^{2} A_{3}^{2}+A_{3}^{2} A_{1}^{2}=\left(A_{1}^{2}+A_{1} A_{2}+A_{2}^{2}\right)^{2}$.

Let us define a vector $\mathbf{A}_{\perp}$ which is the projection of $\hat{\mathbf{A}}$ to the plane containing vectors $\hat{\mathbf{k}}_{1}, \hat{\mathbf{k}}_{2}$, and $\hat{\mathbf{k}}_{3}$. Then the scalar product of these vectors and $\hat{\mathbf{A}}$ is the same as the product with $\mathbf{A}_{\perp}$ :

$$
\hat{\mathbf{A}} \cdot \hat{\mathbf{k}}_{a}=\mathbf{A}_{\perp} \cdot \hat{\mathbf{k}}_{a},
$$

where $a=1,2,3$.

Without loss of generality we can assume that the angle between $\mathbf{A}_{\perp}$ and $\hat{\mathbf{k}}_{1}$ is $\varphi$

$$
A_{1} \equiv \hat{\mathbf{A}} \cdot \hat{\mathbf{k}}_{1}=\mathbf{A}_{\perp} \cdot \hat{\mathbf{k}}_{1}=A_{\perp} \cos \varphi,
$$

where $A_{\perp}=\left|\mathbf{A}_{\perp}\right|$. In equilateral configuration the angle between vectors $\hat{\mathbf{k}}_{1}$ and $\hat{\mathbf{k}}_{2}$ is $2 \pi / 3$, and $A_{2}$ becomes

$$
\begin{aligned}
A_{2} & \equiv \mathbf{A}_{\perp} \cdot \hat{\mathbf{k}}_{2}=A_{\perp} \cos \left(\varphi+\frac{2 \pi}{3}\right) \\
& =-A_{\perp}\left(\frac{1}{2} \cos \varphi+\frac{\sqrt{3}}{2} \sin \varphi\right) .
\end{aligned}
$$

From the last two equations we get

$$
A_{1}^{2}+A_{1} A_{2}+A_{2}^{2}=\frac{3}{4} A_{\perp}^{2} .
$$

Putting this result back into Eq. (A1) we find

$$
\begin{aligned}
A_{1}^{2}+A_{2}^{2}+A_{3}^{2} & =\frac{3}{2} A_{\perp}^{2} ; \\
A_{1} A_{2}+A_{2} A_{3}+A_{3} A_{1} & =-\frac{3}{4} A_{\perp}^{2} ; \\
A_{1}^{2} A_{2}^{2}+A_{2}^{2} A_{3}^{2}+A_{3}^{2} A_{1}^{2} & =\frac{9}{16} A_{\perp}^{4} .
\end{aligned}
$$


[1] E. Komatsu et al., Astrophys. J. Suppl. Ser. 180, 330 (2009).

[2] N.E. Groeneboom and H. K. Eriksen, Astrophys. J. 690, 1807 (2009).

[3] A. A. Starobinsky, Pis'ma Zh. Eksp. Teor. Fiz. 42, 124 (1985).

[4] M. Sasaki and E. D. Stewart, Prog. Theor. Phys. 95, 71 (1996).

[5] D. H. Lyth and Y. Rodriguez, Phys. Rev. Lett. 95, 121302 (2005).

[6] D. H. Lyth, K. A. Malik, and M. Sasaki, J. Cosmol. Astropart. Phys. 05 (2005) 004.

[7] S. Yokoyama and J. Soda, J. Cosmol. Astropart. Phys. 08 (2008) 005 .

[8] K. Dimopoulos, M. Karčiauskas, D. H. Lyth, and Y. Rodriguez, J. Cosmol. Astropart. Phys. 05 (2009) 013.

[9] K. Dimopoulos, Phys. Rev. D 74, 083502 (2006).

[10] K. Dimopoulos, Phys. Rev. D 76, 063506 (2007).

[11] K. Dimopoulos and M. Karčiauskas, J. High Energy Phys. 07 (2008) 119.

[12] M. C. Bento, O. Bertolami, P. V. Moniz, J. M. Mourão, and P. M. Sá, Classical Quantum Gravity 10, 285 (1993).
[13] A. Golovnev, V. Mukhanov, and V. Vanchurin, J. Cosmol. Astropart. Phys. 06 (2008) 009.

[14] L. Ackerman, S. M. Carroll, and M. B. Wise, Phys. Rev. D 75, 083502 (2007).

[15] D. H. Lyth and D. Wands, Phys. Lett. B 524, 5 (2002).

[16] M.S. Turner and L. M. Widrow, Phys. Rev. D 37, 2743 (1988).

[17] T. Moroi and T. Takahashi, Phys. Lett. B 522, 215 (2001); 539, 303(E) (2002).

[18] A. Linde and V. Mukhanov, Phys. Rev. D 56, R535 (1997).

[19] G. Lazarides, R. R. de Austri, and R. Trotta, Phys. Rev. D 70, 123527 (2004).

[20] K. Ichikawa, T. Suyama, T. Takahashi, and M. Yamaguchi, Phys. Rev. D 78, 023513 (2008).

[21] D. H. Lyth, J. Cosmol. Astropart. Phys. 11 (2005) 006.

[22] K. Bamba and J. Yokoyama, Phys. Rev. D 69, 043507 (2004).

[23] K. Bamba and M. Sasaki, J. Cosmol. Astropart. Phys. 02 (2007) 030.

[24] J. Martin and J. Yokoyama, J. Cosmol. Astropart. Phys. 01 (2008) 025.

[25] D. Seery, arXiv:0810.1617. 